\title{
Quand le génome des moustiques fait l'objet d'un projet de séquençage, ou comment lutter contre le paludisme
}

Au début des années 1960, l'éradication du paludisme semblait proche grâce au DDT, un insecticide qui luttait contre la maladie en attaquant les moustiques vecteurs du parasite. Mais, dès la fin de cette décennie, la réalité s'est avérée tout autre. Les moustiques étaient devenus résistants au DDT ainsi qu'à d'autres insecticides et les essais d'inonder les populations d'insectes avec des insectes mâles stériles en vue de diminuer leur taux de reproduction se sont soldés par un échec. La plupart des chercheurs se sont alors tournés vers le parasite luimême et ont cherché à développer des médicaments et des vaccins contre le Plasmodium falciparum. Mais ils se sont rapidement aperçu que celui-ci pouvait devenir résistant aux médicaments et échapper aux vaccins [1].

Actuellement, on s'intéresse à nouveau aux moustiques, non plus pour tenter de les éliminer, mais pour produire des souches transgéniques incapables de transmettre la maladie [2]. L'enjeu est important: le paludisme tue plus d'un million de personnes, surtout des enfants, chaque année en Afrique où il est disséminé par le moustique Anopheles gambiae.

L'équipe de Fotis Kafatos, maintenant directeur général de l'European molecular biology laboratory, vient de publier une carte génétique du chromosome X d'An. gambiae [3]. Cette carte a été réalisée en utilisant les marqueurs microsatellites $(\mathrm{m} / \mathrm{s}$ $n^{\circ} 1$, vol. 9, p. 84), séquences répétées de deux bases et qui ont l'avan- sur le génome, très polymorphes et facilement détectées par PCR. Elle couvre 44 centimorgans pour un total de 31 marqueurs microsatellites localisés à 23 loci. Cette carte a été intégrée au marquage cytogénétique du chromosome et utilisée pour localiser une mutation récessive liée au sexe (œil blanc) entre deux marqueurs espacés de 1 centimorgan. Au cours de l'année, Kafatos pense avoir terminé la carte génétique des deux autres chromosomes d'An. gambiae. L'anophèle n'est pas le seul insecte à faire l'objet d'un projet de séquençage du génome ; une carte génétique de Aedes agypti, l'agent qui transmet la dengue et le virus de la fièvre jaune, vient d'être publiée. Armés de ces cartes, les chercheurs vont ainsi pouvoir localiser très efficacement les traits génétiques des insectes.

Un des premiers indices indiquant que la solution du contrôle du paludisme pouvait résider dans le génome des moustiques date du milieu des années 1980, lorsqu'une souche d'An. gambiae résistante au Plasmodium a été décrite. Cette souche emprisonnait les ookinètes, la forme du parasite qui traverse le mur intestinal du moustique, dans une capsule faite de pigment mélanique. Une manipulation génétique simple, comme le positionnement d'un promoteur fort en amont du gène responsable, pourrait augmenter cet effet contre le parasite. Mais encore fallait-il trouver le gène responsable. C'est peut-être chose faite; Franck Collins, biologiste des vecteurs, au CDC (Center for disease control) d'Atlanta, et Susan Paskewitz, entomologisie à l'université du Wisconsin (USA), ont maintenant cloné un gène candidat codant pour une sérine-protéase qui serait impliquée dans ce processus d'encapsidation. D'autres groupes, dont ceux de Julian Crampton à Liverpool et de Robert Sinden à Londres (GrandeBretagne), ont une approche différente. Au lieu de chercher des gènes de résistance au paludisme chez les anophèles, ils veulent introduire des gènes étrangers au moustique pour le rendre résistant au parasite. Ils ont isolé un gène du système immunitaire des mammifères qui produit un anticorps contre un antigène du Plasmodium porté par l'ookinète. La solution pour que ce gène ne s'exprime qu'au bon moment et au bon endroit pourrait venir du groupe de Crisanti à Rome (Italie) [4] qui a séquencé chez $A n$. gambiae deux gènes codant pour des enzymes digestives appartenant à la famille des trypsines. Ces gènes ne s'expriment au niveau de l'intestin du moustique que lors du repas sanguin. Il faudrait alors lier leurs séquences promotrices au gène produisant l'anticorps contre le Plasmodium, et il y aurait libération au niveau intestinal d'une grande quantité d'anticorps qui se fixerait sur les ookinètes absorbés lors du repas sanguin.

Avant que cette théorie particulièrement séduisante ne puisse être testée, il faudrait être capable d'insérer des gènes étrangers chez le moustique afin d'obtenir des insectes transgéniques. Les travaux effectués sur la mouche tsé-tsé (Clossina morsitans), agent de la trypanoso- 
miase africaine, offrent une alternative intéressante. En effet, ces insectes ont en symbionte des bactéries à l'intérieur des cellules de leur intestin ou d'autres tissus, et l'on pourrait créer des mouches tsé-tsé "pseudotransgéniques " en injectant le gène, non dans la mouche, mais dans le symbionte. Bien que $A n$. gambiae ne possède pas ce type de bactérie, certains chercheurs testent si Wolbachia pipientis, une bactérie vivant dans les ovaires des mouches tsé-tsé, pourrait être introduite chez l'anophèle [1]. D'autres prennent une voie plus classique en utilisant, soit des rétrovirus, soit des éléments transposables, du type des éléments $\mathrm{P}$, fonctionnels chez la drosophile. H. Robertson, de l'université d'Illinois (USA) travaille, quant à lui, sur une famille d'éléments transposables appelés mariner, présents chez de nombreux insectes [5]. An. gambiae possède un mariner proche de celui de certaines mouches, mais ces mariner semblent avoir perdu leur capacité de transposition. C'est pourquoi cette équipe a ajouté à cet élément transposable une séquence promotrice puissante, espérant ainsi lui redonner le pouvoir de se mobiliser.

En attendant que les chercheurs arrivent à créer des insectes transgéniques, plusieurs gènes candidats anti-Plasmodium auront peut-être été isolés. Le but final serait de créer des moustiques transgéniques avec plusieurs gènes afin de diminuer les risques de résistance du parasite. Il faudra bien sûr s'assurer de l'absence de danger d'une telle approche avant de lancer dans la nature un grand nombre d'insectes transgéniques. Il s'agit d'un programme échelonné sur quinze à vingt ans, a déclaré Tore Godal, le responsable des maladies tropicales à l'OMS, mais sur lequel il est possible de fonder de réels espoirs [1].

E.D.

1. Aldhous P. Malaria: focus on mosquito genes. Science 1993; 261: 546-8.

2. James AA. Mosquito molecular genetics: the hands that feed bite back. Science 1992 ; $257: 37-8$.

3. Zheng L, Collins FH, Kumar V, Kafatos $\mathrm{FC}$. A detailed genetic map for the $\mathrm{X}$ chromosome of the malaria vector, Anopheles gam bice. Science 1993; 261: 605-8.

4. Muller HM, Crampton JM, della Torre A Sinden R, Crisanti A. Members of a trypsin family in Anopheles gambice are induced in the gut by blood meal. $1: M B()$ J 1993; 12 : $2891-900$

5. Robertson HM. The mariner transposable element is widespread in insects. Nature 1993 362: 241-5.

\section{BRÈVES IIIT}

Localisation chromosomique et clonage du gène de la maladie de Krabbe. La maladie de Krabbe ou leucodystrophie à cellules globoïdes est une affection neurologique à hérédité récessive autosomique due au déficit en galactocérébrosidase (GALC). Ce déficit empêche la dégradation du galactosylcérébroside; il est d'une haute gravité et conduit à la mort en général dans les deux premières années. C'est une des rares lipidoses dont le gène n'avait pas encore été cloné. L'équipe de W'enger (Philadelphie, PA, USA) a fait coup double en décrivant, dans deux articles séparés, et la localisation chromosomique et le clonage de l'ADNc. Le siège du gène sur le chromosome 14, déjà suggéré en 1990 [1], était probable parce qu'un déficit en GALC de la souris, mutation "twitcher", est sur le chromosome 12, dans une région homologue du 14 humain. Associée à des cher- cheurs de Jérusalem, l'équipe de Philadelphie a étudié [2] seize familles par analyse de liaison, montrant une localisation sur le bras long, en 14q24-q32 (lod score supérieur à 13) $\left(\mathrm{m} / \mathrm{s} n^{\circ} 12\right.$, vol. 9, p. 1418). Un déséquilibre de liaison entre le locus de la maladie et un marqueur voisin a été, en outre, découvert dans une communauté druze en Israël. La recherche du gène [3] est partie d'une fraction purifiée de (iALC: de cerveau, dont la portion $\mathrm{N}$-terminale a pu être séquencée. Des amorces nucléotidiques dérivées ont été utilisées pour amplifier de l'ARN de testicule de chat, tissu particulièrement riche en GALC. Le produit obtenu a servi à cribler des banques de testicule et de cerveau humain. Les clones ainsi isolés ont permis l'analyse de la région codante et la déduction de la séquence des 669 acides aminés, dont 26 pour un peptide signal. La transfection de
l'ADNc en cellules COS a montré l'apparition d'une activité ('ALC, toutefois très faible, et qui pouvait être notablement amplifiée en modifiant la séquence qui entoure le codon d'initiation ; celle-ci est en effet peu favorable à une expression forte sous forme native, ce qui explique peut-être la faible activité de l'enzyme dans les tissus normaux.

L'obtention de ce clone est un premier pas dans l'analyse moléculaire de la maladie, prometteur de progrès rapides, car plusieurs modèles animaux existent (souris mais aussi chat, chien, mouton), qui pourront être exploités pour des tentatives thérapeutiques.

[1. Zlotagora J, el al. Am J Hum (ienet 1990; 47: 37-44.]

[2. Oehlmann R, et al. Am I Hum (ienet 1993, 53 : 1250-5.]

[3. Chen YQ et al. Hum Mol (ienet 1993, 2: 1841-5.]

(993, 2: $1841-5.1$

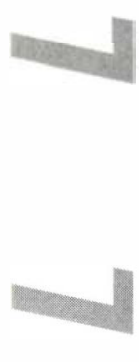

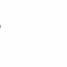

